\title{
A proposito di due lettere inedite della Contessa d'Albany a Sismondi
}

\section{Francesca Piselli}

\section{(2) OpenEdition}

1 Journals

\section{Edizione digitale}

URL: https://journals.openedition.org/studifrancesi/2952

DOI: 10.4000/studifrancesi.2952

ISSN: $2421-5856$

\section{Editore}

Rosenberg \& Sellier

\section{Edizione cartacea}

Data di pubblicazione: 1 juillet 2013

Paginazione: 321-334

ISSN: 0039-2944

\section{Notizia bibliografica digitale}

Francesca Piselli, «A proposito di due lettere inedite della Contessa d'Albany a Sismondi», Studi

Francesi [Online], 170 (LVII | II) | 2013, online dal 30 novembre 2015, consultato il 02 février 2023. URL: http://journals.openedition.org/studifrancesi/2952 ; DOI: https://doi.org/10.4000/studifrancesi.2952

\section{(c) (i) $\odot$}

Creative Commons - Attribuzione - Non commerciale - Non opere derivate 4.0 Internazionale - CC BYNC-ND 4.0

https://creativecommons.org/licenses/by-nc-nd/4.0/ 


\title{
A proposito di due lettere inedite della Contessa d'Albany a Sismondi
}

\begin{abstract}
Two letters from Louise Stolberg Gedern, Countess of Albany to Jean-Charles-Léonard Simonde de Sismondi are herein edited and presented. They reveal some still unknown details not only about the friendship between the famous letter writer and the Genevan historian, but also about their relationships with Madame de Staël, a mutual friend, and other members of the Coppet group. Moreover, contemporary political issues are also debated. In particular, the two correspondents express different opinions about Napoleon. These letters, showing specific orthographical, morpho-syntactical and lexical features, represent a remarkable example of French as universal communication language at the beginning of the XIX ${ }^{\text {th }}$ century.

Deux lettres inédites de Louise Stolberg Gedern, Comtesse d'Albany à Jean-Charles-Léonard Simonde de Sismondi sont ici éditées et présentées. Elles révèlent des détails, jusqu'alors inconnus, sur l'amitié entre la célèbre épistolière et l'historien genevois, sur leurs rapports avec Madame de Staël, amie commune, aussi bien qu'avec plusieurs personnages liés au groupe de Coppet. La situation politique de l'époque y est également reportée. La discussion des deux correspondants se concentre notamment autour de la figure de Napoléon, qui est loin de faire l'unanimité entre eux. Ces lettres, présentant des particularités aux niveaux orthographique, morpho-lexical, syntaxique, aussi bien que lexical, se signalent comme un exemple remarquable de l'usage du français comme langue de communication au début du XIX siècle.
\end{abstract}

Le due lettere qui presentate sono una testimonianza dell'amicizia che legava Louise Stolberg-Gedern, Contessa d'Albany (Mons 1752 - Firenze 1824) ${ }^{1}$, instanca-

(1) La vita di Louise Maximiliana Carolina Emanuela Stolberg-Gedern, questo il nome completo, ebbe contorni romanzeschi e ha offerto numerosi spunti ai suoi tanti biografi. Tra le principali biografie a lei dedicate, si vedano A. von Reumont, Die Gräfin von Albany, Berlin, Verlag der Königlichen Geheimen Ober-Hofbuchdruckerei (R. Decker), II Bd., 1860 (trad. it. di A. DI Cossilla, La contessa d'Albany, Genova, co' tipi del R. I. de' Sordo-Muti, 1868); S.-R. TAILLANDIER, La comtesse d'Albany, Paris, Lévy, 1862; L.-J. Thomas, Une femme, son roi, son poète et son peintre, Montpellier, Librairie Coulert, 1928; A. MAURIAC, Trois prortraits de femmes: La duchesse de Devonshire, la comtesse d'Albany, Henriette-Marie de France, Paris, Hachette, 1967 C. Pellegrini, La contessa d'Albany e il salotto del Lungarno, Napoli, Edizioni Scientifiche Italiane, 1951; M. Crosland, Louise of Stolberg, Countess of Albany, Edinburgh-London, Oliver and Boyd, 1962. A queste si aggiunge la recente biografia di A. DE LACRETELLE, La comtesse d'Albany. Une égérie européenne, Paris, Edition du Rocher, 2008. Da segnalare anche la ristampa di un'altra biografia otto- centesca, vale a dire V. LEE (pseudonimo di Violet Paget), The countess of Albany, Boston, Roberts Brothers, 1884 ( $2^{\mathrm{a}}$ ed.: London, John Lane, 1909), Teddington, The Echo Library, 2009. Sulla vita della contessa d'Albany, si veda anche il romanzo della tedesca Amalie Bölthy, dal titolo: Vittorio Alfieri ovvero Torino e Firenze nel secolo XVIII. Romanzo storico, trad. it. di G. Stafforello, Milano, Editori della Biblioteca Utile, 1866. Per una rassegna delle biografie dedicate alla Stolberg, cfr. L. RICALDONE, La donna «nuova» e il «genio»: per un ritratto di Luisa Stolberg, in Alfieri e il suo tempo, Atti del Convegno internazionale, Torino - Asti 29 novembre - 1 dicembre 2001, a cura di M. Cerruti, M. Corsi, B. Danna, Firenze, Olschki, 2003 , pp. 323-342. Accenni alla contessa d'Albany anche in P. L. COURIER, Conversation chez la comtesse d'Albany, in Euvres Complètes de P. L. Courier, nouvelle édition, Paris, Didot, 1839, pp. 407-415; E. e J. DE GonCourT, La Comtesse d'Albany, in Portraits intimes du dix-buitième siècle, Paris, Charpentier, 1878, pp. 425-461; C. A. Sainte-Beuve, Nouveaux Lundis, XII vol., Paris, Calmann Lévy, 1890-1899, V, pp. 395-439; 
bile épistolière e nota salonnière dalla cultura cosmopolita, a Jean-Charles-Léonard Simonde de Sismondi (Genève 1773 - 1842), membro del gruppo di Coppet, apprezzato economista, storico e critico letterario ginevrino ${ }^{2}$. Entrambe autografe e inedite, esse fanno parte del ricco Fondo Sismondi, depositato presso l'Archivio di Stato di Pescia. Tale fondo si compone di tre versamenti successivi e si segnala per la presenza di ben 1.700 lettere femminili, ossia quasi la metà dell'intero carteggio ${ }^{3}$.

Gli originali qui pubblicati, firmati dalla Contessa, sono indirizzati appunto al Sismondi, rispettivamente in data 23 giugno 1807 e 21 maggio 1814. Essi appartengono al secondo versamento e non fanno parte del corpus di 65 lettere della Contessa a Sismondi, dato alle stampe da Carlo Pellegrini nel 1951. A questo studioso si deve pure la pubblicazione dell'Epistolario dello storico ginevrino, il cui primo volume apparve nel $1933^{4}$.

Stendhal (H. Bayle), Voyage en France, in Voyages en France, Paris, Gallimard, Bibliothèque de la Pléiade, 1992, p. 550; ID., Voyage dans le Midi de la France, in op. cit., pp. 697-703; ID., Rome, Naples et Florence, Paris, Gallimard, Bibliothèque de la Pléiade, 1973 , p. 74 , p. 490, p. 701; F.-R. DE CHATEAUBRIAND, Mémoires d'outre-tombe, Paris, Gallimard, Bibliothèque de la Pléiade, 1951, vol. II, pp. 248-249; S. Owenson (LADY Morgan), L'Italie, t. IV, Paris, P. Dufart Libraire, 1821, t. III, pp. 6366; M. D'Azeglio, I miei ricordi, Torino, Utet, 1971, pp. 123-126.

(2) Si ricorda che Sismondi, dopo aver viaggiato in Francia e in Inghilterra, fu costretto a fuggire in Italia per motivi politici. Si stabilì in Toscana, terra dei suoi avi, dove la famiglia Sismondi acquistò, nel 1794, la villa di Valchiusa, vicino Pescia. Durante la sua vita alternò soggiorni nella tenuta pesciatina a periodi all'estero, in particolare a Ginevra. Per un profilo biografico di Sismondi, cfr. J.-R. De Salis, Sismondi (1773-1842). La vie et l'ouvre d'un cosmopolite philosophe, Paris, H. Champion, 1932; P. WAEBER, Sismondi. Une biographie, I, Les devanciers et la traversée de la Révolution. Chroniques familiales (1692-1800), Genève, Slatkine, 1991; L. GILlaRD, Simonde de Sismondi, vie, auvres, concepts, Paris, Ellipses Marketing, 2010 e relativa bibliografia. Su Sismondi e la Toscana, visti i numerosi studi esistenti, ci limitiamo a rimandare a Sismondi e la civiltà toscana, Atti del Convegno internazionale di studi, Pescia, 13-15 aprile 2000, Firenze, L.-S. Olschki, 2001, con appendice bibliografica a cura di L. Pagliai; C. Pazzagli, Sismondi e la Toscana del suo tempo (1795-1838), Siena, Protagon Editori Toscani, 2003, pp. 278; Sismondiana. In onore di Mirena Stanghellini Bernardini, a cura di L. Pagliai, Firenze, Polistampa, 2005; A. G. RICCI, Esercizi sismondiani, 1970-2005, a cura di L. Pagliai, Firenze, Edizioni Polistampa, 2008.

(3) Il primo versamento (1931) fu fatto dalle ultime eredi dirette, le signorine Desideri, che avevano donato al comune $\mathrm{l}$ ampio carteggio di $\mathrm{Si}$ smondi ( 25 scatole contenenti 3953 lettere) e altre 24 scatole contenenti 118 fascicoli di manoscritti e documenti diversi sempre dello scrittore. Con il secondo versamento (1967), il Comune di Pescia ebbe anche le carte considerate più propriamente familiari, tra le quali si trovano i Diari della madre e della sorella, oltre alle lettere dei loro corrispondenti. Il terzo versamento (1971) ha aggiunto un nucleo di 64 lettere dello scrittore e alcune sue minute, donate al Comune per arricchire il fondo. Quest'ultimo, conservato fino a pochi anni fa presso la Biblioteca Comunale di Pescia, di recente è stato spostato nella locale sezione dell'Archivio di Stato di Pescia. Sull'archivio Sismondi, si vedano M. Bernardini Stanghellini, L'archivio Sismondi nella Biblioteca Civica di Pescia, in A.G. RIcci, L'archivio Sismondi, in «Archivi e Cultura», XIII, 1979, pp. 103-140; M. ChIOsTRI, La famiglia Forti di Pescia attraverso l'epistolario di J.C.L. Simonde de Sismondi, in «Il Viesseux», 5, 1989, pp. 16-24; I. PERA, Scritture femminili nei fondi d'archivio delle province di Lucca e Pistoia, in Carte di donne. Per un censimento regionale della scrittura delle donne dal XVI al XX secolo, vol. II, «Atti della giornata di studio», Firenze, Archivio di Stato, 3 febbraio 2005, a cura di A. Contini e A. Scattigno, Roma, Edizioni di Storia e Letteratura, 2007, pp. 229-238. Per quanto riguarda le voci femminili nel fondo Sismondi, cfr. M. P. CASALENA, Amicizie epistolari. Ruoli di genere e percorsi biografici nel Fondo Sismondi, in "Cher Sis.". Scritture femminili nella Corrispondenza di Sismondi, a cura di M. P. Casalena e F. Sofia, Firenze, Polistampa, 2008, pp. 7-60.

(4) Cfr. J.-C.-L. Sismond, Epistolario, a cura di Carlo Pellegrini, 4 voll. Vol. I (1799-1814), Firenze, La Nuova Italia,1933; vol. II (1814-1823), Firenze, La Nuova Italia, 1935; vol. III (1824-1835), Firenze, La Nuova Italia, 1936; vol. IV (18361842), Firenze, La Nuova Italia, 1954. Alcune lettere di Sismondi alla contessa d'Albany, seppure non in versione integrale, erano apparse precedentemente in Lettres inédites de J.C.L. Sismondi, de M. de Bonstetten, de Madame de Staël, de Madame de Souza à Madame la Comtesse d'Albany publiées avec une introduction par M. Saint-René Taillandier, Paris, Michel Lévy, 1865. Le citate lettere della contessa d'Albany a Sismondi sono pubblicate in appendice al volume C. PellegrinI, $\mathrm{La}$ contessa d'Albany, cit., pp. 279-365. Sull'epistolario di Sismondi, si veda anche L. E. Funaro, Mes deux patries: minime aggiunte all'epistolario del Sismondi, in «Archivio storico italiano», n. 593/3, 2002, pp. 556-588. 
Lo scambio di idee, a tratti anche vivace, tra la regina in partibus e Sismondi è in parte noto. Queste lettere tuttavia aggiungono ulteriori, interessanti dettagli sul loro legame d'amicizia, nonché sui punti di vista dei due corrispondenti riguardo alle complesse vicende politiche di quegli anni.

La lingua delle lettere di questa «hôtesse européenne» è stata spesso criticata dagli editori del XIX e degli inizi del XX secolo, che hanno tentato di modernizzarla e di normalizzarla dal punto di vista ortografico, oltre che nell'uso degli accenti, degli apostrofi e della punteggiatura ${ }^{5}$. Come è noto, il francese fu la lingua della sua educazione e, benché durante i lunghi anni trascorsi a Firenze avesse imparato l'italiano, la Contessa preferì usare la lingua francese sia con gli ospiti che frequentavano il suo salotto, sia con Vittorio Alfieri ${ }^{6}$. È altresì vero che il francese, per diretta ammissione di questa celebre épistolière, costituiva «le moyen de dire plus facilement» ciò che voleva dire, senza alcuna pretesa di eleganza ${ }^{7}$. Louise Stolberg scriveva praticamente di getto: «je vous communique mes idées», spiegava al tesoriere dell'Università di Siena, Alessandro Cerretani, «comme elles se présentent sans art et sans prétention». La finalità principale di queste lettere, come suggerisce a più riprese la nobildonna stessa, era semplicemente quella di farsi capire per restare in contatto con persone di varia provenienza linguistica.

Per quanto ci riguarda, concordiamo pienamente con quanto suggerito a suo tempo da Carlo Pellegrini. Questi sostiene che il francese della comtesse è «il francese del cosmopolita» e che, se studiato nella sua veste originale, può fornire un paragone non trascurabile alla descrizione di questa lingua ${ }^{9}$, come lingua franca di comunicazione nel delicato tournant tra XVIII e XIX secolo. La lingua della Contessa sembra riflettere questo passaggio e appare in bilico tra il rispetto degli usi classici (prevalente peraltro nelle lettere fino al 1810 circa), e l'introduzione d'innovazioni ortografiche, morfologiche, sintattiche e lessicali, soprattutto nelle lettere della maturità.

Tenuto conto di ciò, abbiamo preferito adottare nella trascrizione un criterio strettamente conservativo. Le peculiarità della lingua della Contessa d'Albany sono state segnalate in nota. Inoltre le parole scritte nell'interlinea sono state riportate tra parentesi uncinate. Infine i casi in cui si è reso necessario il ricorso allo scioglimento di abbreviazioni o a qualche congettura interpretativa sono stati indicati tra parentesi quadre.

L'originale della prima lettera consta di quattro facciate. L'indirizzo compare all'esterno e reca il sigillo della Contessa su ceralacca rossa. Il testo occupa due facciate e mezzo, che si compongono rispettivamente di ventiquattro righe la prima, di ventitré la seconda e di quindici la terza.

Questa lettera ci consente di anticipare di diversi mesi l'inizio della corrispondenza tra la nobildonna e Sismondi, rispetto a quanto noto finora ${ }^{10}$. Il dialogo epi-

(5) Per quanto concerne i problemi linguistici, ci permettiamo di rimandare al nostro F. PISELLI, Lettere inedite della contessa d'Albany alla National Library of Scotland, in «Annali della Facoltà di Lettere e Filosofia dell'Università degli Studi di Perugia. Studi linguistico letterari», vol. XLI, n. s. XXVIII, A.A. 2004/2005, pp. 263-282.

(6) V. Alfieri, Vita, a cura di A. Dolfi, Milano, Mondadori, 2004 (I ed. 1987), p. 244.

(7) Lettres inédites de la comtesse d'Albany à ses amis de Sienne (1797-1820), mises en ordre et publiées par L.-G. Pélissier. Tome I. Lettres à Teresa Regoli Mocenni et au chanoine Luti (1797-1802), Paris, Fontemoing, 1904; tome II. Lettres à l'archiprêtre Luti et à Vittorio Mocenni (1802-1809), Toulouse, E. Privat, 1912 tome III. Lettres à Alessandro Cerretani (1803-1820),
Toulouse, E. Privat, 1915, t. III, p. 5 . Il 9 aprile 1808 , la Contessa scriveva a Cerretani: «Je n'ai jamais eu la vanité de briller par mon style» (Lettres inédites, cit., t. III, p. 91). Cfr. anche la ristampa dei primi due tomi: Lettres inédites de la Comtesse d'Albany à ses amis de Sienne, publiées par Léon-G. Pélissier (1797-1802), ristampa a cura di R. Turchi, Firenze, Società Editrice Fiorentina, 2009.

(8) Lettres inédites, cit., t. III, p. 91.

(9) C. Pellegrini, La contessa d'Albany, cit., p. 46.

(10) La prima lettera della Stolberg a Sismondi, pubblicata da Pellegrini, è datata 22 marzo 1808 (cfr. C. Pellegrini, La contessa d'Albany, cit., p. 279). 
stolare tra i due letterati durò fino alla morte di lei ${ }^{11}$. La Contessa aveva conosciuto Sismondi nel 1805; era stata Madame de Staël, durante il suo primo viaggio in Italia, a introdurlo a Palazzo Gianfigliazzi, residenza fiorentina della principessa Stolberg.

L'autore dell'Histoire des Français nutriva una profonda ammirazione verso Vittorio Alfieri e, inizialmente, fu proprio nel suo nome che strinse amicizia con Madame d'Albany. Sismondi apprezzava le doti di ascoltatrice della Contessa, forse apprese negli anni trascorsi accanto al poeta astigiano. L'immagine che il ginevrino aveva di quest'ultima era fortemente influenzata dall'idealizzazione fattane dalla «Dame chatelaine» ${ }^{12}$. La Stolberg rappresentava agli occhi dell'amica il prototipo dell'égérie - come è stato scritto in una recente biografia a lei dedicata -, oltre che la custode della produzione e della memoria alfieriane ${ }^{13}$.

Il primo autografo, datato 23 giugno 1807, si apre con il riferimento alla pubblicazione del tomo iniziale dell'Histoire des Républiques italiennes au Moyen Âge di Sismondi, avvenuta proprio in quell'anno ${ }^{14}$. Secondo quanto emerge da una lettera del 18 giugno 1807, questi aveva inviato i primi due volumi dell'opera a Madame d'Albany, in segno di rispetto per la 'vedova' del poeta da lui tanto ammirato:

Si votre noble ami avait vécu, c'est à lui que j'aurais ambitionné d'obtenir [l'approbation] par-dessus tous les autres. Son âme, généreuse et fière, appartenait à ces siècles de grandeur et de gloire, que j'ai cherché à faire connaître. Né comme par miracle hors de son siècle, il appartenait tout entier à des temps qui ne sont plus, et il avait donné à l'Italie comme un monument de ce qu'avaient été ses enfants, comme un gage de ce qu'ils pouvaient être encore. Il me semble que l'amie d'Alfieri, celle qui consacre désormais sa vie à rendre un culte à la mémoire de ce grand homme, sera prévenue en faveur d'un ouvrage d'un de ses plus zélés admirateurs, d'un ouvrage où elle retrouvera plusieurs des pensées et des sentiments qu'Alfieri a développés avec tant d'âme et d'éloquence ${ }^{15}$.

Tuttavia ad avvicinare Sismondi alla Contessa contribuì anche la comune ostilità nei confronti di Napoleone. Per essere più precisi, tale antipatia era notoriamente condivisa da tutti i membri del gruppo di Coppet e, prima fra tutti, da Madame de Staë ${ }^{16}$. Non manca, nella lettera della compagna di Alfieri, il riferimento alla scrittrice

(11) È bene precisare che le divergenze tra la Contessa d'Albany e Sismondi furono legate a questioni di natura politica, come avremo modo di rilevare più avanti. Dopo il 1814 la loro corrispondenza fu meno regolare. Delle citate 65 lettere pubblicate da Pellegrini, solo 8 appartengono al periodo 1816-1823; un'unica lettera risale al 1815. Durante i "Cento giorni", con l'aperto sostegno di Sismondi a Napoleone, ci fu una rottura quasi completa con la Stolberg. Il carteggio riprese nel 1816, anche se le incomprensioni non furono mai del tutto superate. Cfr. ivi, p. 148.

(12) Appellativo spesso usato nelle lettere di Louise Stolberg per riferirsi a Madame de Staël (C. Pellegrini, La contessa d'Albany, cit., p. 281). Lo stesso Pellegrini riporta le parole della Staël a proposito di Madame d'Albany: «J'ai été constamment attendrie et de Madame d'Albany et de cette maison où Alfieri a vécu, aimé, souffert. (...). Mme d'Albany n'a pas beaucoup d'esprit, mais du naturel et ses yeux sont toujours remplis de larmes en le nommant. Il lui a dit avant de mourir: Ma chère amie, si l'on ne me guérit pas, il faudra donc nous séparer. J'ai été bien attendrie par la douleur de Mme d'Albany» (G. Reval, Le secret de Corinne.
Oswald et le duc de Palmella, «Occident et Cahiers Staëliens», t. II, n. 3, 15 février, 1935, pp. 205-213, cit. in ivi, p. 144).

(13) A. DE LACretelle, La comtesse d'Albany, cit., p. 271.

(14) J.-C.-L. Sismond, Histoire des Républiques Italiennes du Moyen Age, XVI, 8 voll., Paris, H. Nicolle, puis Treuttel et Würtz, 1807-1818. Cfr. C. Cordié, Contributo bibliografico sul gruppo di Coppet. Scritti di Mme de Staël, Sismondi, B. Constant, Ch. $-V$. de Bonstetten apparsi in Italia o in lingua italiana dal 1803 al 1983, in «Annali della Scuola Normale Superiore di Pisa», t. XXI, n. 2, 1991, pp. 695-735; P. SCHIERA, Presentazione, in J.-C.-L. Sismond, Storia delle Repubbliche italiane, Torino, Bollati Boringhieri, 1996; P. A. BorgHEGGIANI, Sur quelques traductions italiennes d'ouvrages historiques de Sismondi, in Ordre et caprice. De Bonstetten à Bourget, Fasano-Paris, Schena-Presses de l'Université de Paris-Sorbonne, 2002, pp. 22-34.

(15) Lettres inédites de J.C.L. Simondi, cit., pp. 67-68. La stessa lettera è stata ripubblicata in J.-C.-L. Sismondi, Epistolario, cit, vol. I, p. 152.

(16) Sulla vita della Baronessa de Staël, si veda il recente M. WINOCK, Madame de Staël, Paris, Fa- 
di origini svizzere e segnatamente al romanzo Corinne ou l'Italie, apparso tra la fine di aprile e i primi di maggio del $1807^{17}$. La Stolberg non lo aveva ancora ricevuto, nonostante la Staël avesse provveduto a inviarglielo; come scrive Sismondi nella risposta del 25 giugno, «c'est à vous que l'auteur voulait que son livre parvînt avant tout autre en Italie» ${ }^{18}$.

La Contessa d'Albany attendeva con impazienza di leggere Corinne per vedere cosa si dicesse sul poeta, soprattutto dopo il giudizio non del tutto lusinghiero espresso in De la littérature, altra opera della bôtesse di Coppet, apparsa nel $1800^{19}$. Malgrado qualche riserva sull'unilateralità dei personaggi e sulla monotonia delle tragedie, la Staël rivela in Corinne un «respect profond pour le caractère d'Alfieri» ${ }^{20} \mathrm{e}$ sottolinea il contrasto fra la grandezza d'animo dell'Astigiano e il momento storico in cui visse ${ }^{21}$. Sismondi, nella lettera del 18 giugno 1807, fa eco alle parole di Madame de Staël, asserendo che lo spirito generoso e fiero di Alfieri era del tutto estraneo al secolo in cui era nato ${ }^{22}$.

Dopo aver letto il romanzo in questione, la Contessa d'Albany espresse il suo giudizio e ravvisò il tratto principale dell'opera nell'oscillazione tra l'essere libro di viaggio e romanzo:

Je la regarde [Corinne ou l'Italie] comme son meilleur ouvrage. Il y a des détails charmants, de jolies descriptions; elle y peint très bien les Italiens, les Français et les Anglais. Elle se trompe souvent, dans le cours du voyage d'Italie, sur les objets d'art, mais ce n'est pas un voyage qu'elle veut faire; le voyage n'est que le prétexte. L'ensemble du livre n'est pas digéré; mais malgré cela, cet ouvrage est intéressant ${ }^{23}$.

Nell'autografo della Principessa Stolberg qui pubblicato si fa allusione pure all'«aventure» dell' autrice di Delphine che, nonostante l'interdizione da Parigi, aveva avuto il permesso di acquistare «une campagne dans la vallée de Montmorency» ${ }^{24}$, a nord-ovest della capitale, salvo poi impedirle di abitarvi. Oltre all'attrazione da sempre esercitata su di lei da Parigi, la Baronessa de Staël intendeva avvicinarsi alla capitale per sovrintendere alla pubblicazione di Corinne. Con grande disappunto, fu costretta a riprendere la via di Coppet nei giorni immediatamente precedenti l'apparizione del romanzo.

yard, 2010, a cui si rinvia anche per la bibliografia antecedente.

(17) Mémoires de Mme de Staël. Dix années d'exil. Ouvrage postbume publié en 1818. Nouvelle édition, précédée d'une notice sur la vie et les ouvrages de Mme de Staël par M. le duc de Broglie et M. le Bon de Staël, Paris, Charpentier, 1861, pp. 505-506. Cfr. C. Pellegrini, Il Sismondi e Napoteone, in Letteratura e storia nell'Ottocento francese e altri saggi, Roma, Edizioni di Storia e Letteratura, 1967, p. 11.

(18) J.-C.-L. Sismondi, Epistolario, cit., vol. I, pp. 153-154. Cfr. Lettres inédites de J.C.L. Sismondi, cit., p. 68.

(19) Cfr. G. DE STAËL-Holstein, De la littérature, édition établie par G. Gengembre et J. Goldzink, Paris, Flammarion, 1991, p. 160.

(20) G. DE StaËL-Holstein, Euvres complètes, série II. Euvres littéraires, tome III, Corinne ou l'Italie, édition critique par S. Balayé, Paris, H. Champion, 2000, p. 223.

(21) Madame de Staël scriveva: «Alfieri, par un hasard singulier était, pour ainsi dire, transplanté de l'antiquité dans les temps modernes, il était né pour agir, et il n'a pas pu qu'écrire: son style et ses tragédies se ressentent de cette contrainte (...). Alfieri, impatienté de vivre au milieu d'une nation où l'on rencontrait des savants très érudits et quelques hommes très éclairés, mais dont les littérateurs et les lecteurs ne s'intéressaient pour la plupart à rien de sérieux, et se plaisaient uniquement dans les contes, dans les nouvelles, dans les madrigaux; Alfieri, dis-je, a voulu donner à ses tragédies le caractère le plus austère. (...) il a pourtant été fort admiré, parce qu'il est vraiment grand par son caractère et par son âme (...). Mais il n'en est pas moins vrai qu'Alfieri n'a pas créé ce qu'on pourrait appeler un théâtre italien, c'est-à-dire des tragédies dans lesquelles on trouvât un mérite particulier à l'Italie» (ibidem). Cfr. C. Pellegrini, La contessa d'Albany, cit., pp. 150-151.

(22) J.-C.-L. Sismond, , I, p. 152. Cfr. Lettres inédites de J.C.L. Simondi, cit., p. 67.

(23) Louise Stolberg a Alessandro Cerretani, 15 settembre 1807, in Lettres inédites, cit., t. III, pp. 73-74.

(24) Lettres inédites de J.C.L. Simondi, cit., p. 153. 
La Contessa d'Albany parla di ostinazione in questo voler tornare a Parigi, perché, sempre a suo dire, l'amica non può fare a meno della «futilité» della vita mondana, dimostrando mancanza di forza di carattere. Sismondi, nella risposta del 25 giugno, cerca di sfumare il giudizio netto della Stolberg e, pur concordando sul fatto che la scrittrice francese manchi di «fermeté dans le caractère» ${ }^{25}$, suggerisce che «elle est attirée vers cette ville, qui est sa patrie, par des liens plus forts que ceux de la societé» ${ }^{26}$. Ad ogni modo la Comtesse aggiunge anche che proprio questo lato del carattere rende l'amica francese più umana. Qui il pensiero e il paragone vanno, come spesso accade nei suoi carteggi, a Vittorio Alfieri, figura ideale di uomo, che univa la dolcezza, la forza d'animo e la fermezza al talento.

Un altro aspetto interessante della lettera è l'accenno all'edizione delle Opere Postume del poeta astigiano. La Stolberg si era assunta tale compito al momento della morte del compagno, avvenuta nel 1803. Alfieri aveva dettato le sue ultime volontà alcuni anni prima, nel 1799, sottolineando che si trattava di «semplici preghiere da eseguirsi o no, secondo le circostanze e il total piacimento della Contessa ${ }^{27}$. In realtà quest'ultima prese molto a cuore l'impegno e affidò allo stampatore fiorentino Guglielmo Piatti i manoscritti alfieriani, cedendo la proprietà editoriale, ma riservandosi il diritto di rivedere accuratamente le bozze ${ }^{28}$.

Nell'autografo sotto trascritto, Madame d'Albany sostiene di essere quasi alla fine dell'impresa, mancando solo la Vita al resto delle opere ${ }^{29}$. Si tratta di un'affermazione di non poco conto, viste le difficoltà incontrate dal progetto. Nel settembre 1806 era giunta l'interdizione delle autorità borboniche, a cui era seguito l'ordine di confisca. Il 28 febbraio 1807 la Contessa annunciava ad Alessandro Cerretani, con un certo sollievo, che era stato tolto il sequestro e che finalmente la pubblicazione poteva essere portata a termine ${ }^{30}$. Tuttavia alla fine di aprile restava il divieto di vendita delle copie, anche se alcune, soprattutto tra gli amici, circolavano clandestinamente ${ }^{31}$. Nell'inedito qui presentato, si fa riferimento alla proibizione delle Commedie. È comunque noto che alcune riproduzioni non autorizzate delle Postume furono effettuate dagli stampatori milanesi Agnelli (1804) e Silvestri (1806) ${ }^{32}$, oltre che in molte altre parti d'Italia, a riprova della limitata efficacia della censura che danneggiò l'impresa della Contessa d'Albany, ma non la bloccò. Quest'ultima andò avanti, dicendosi sicura che il tempo avrebbe reso giustizia al lascito alfieriano.

Nella parte finale della lettera la Stolberg accenna a un altro personaggio legato al gruppo di Coppet, vale a dire il letterato, filosofo e viaggiatore svizzero Charles Victor de Bonstetten (Berne 1745 - Genève 1832). La regina in partibus aveva stretto una tenera amicizia con Bonstetten nel 1774, quando viveva a Roma con il marito. Nell'autunno di quell'anno la coppia regale partì alla volta della Toscana. La Contessa ebbe occasione di ritrovare Bonstetten a Siena; poi, una volta stabilitasi a Firenze, continuò lo scambio epistolare con il bernese per circa un anno ${ }^{33}$. Quest'ultimo an-

(25) Ivi, p. 154.

(26) Ibidem.

(27) V. Alfieri, Vita scritta da esso, Asti, Casa d'Alfieri, 1951, vol. II, p. 310.

(28) M. PASTA, Guglielmo Piatti editore di Alfieri, in AA. Vv., Alfieri in Toscana, Atti del Convegno Internazionale di Studi (Firenze, 19-21 ottobre 2000), a cura di G. Tellini e R. Turchi, Firenze, Olschki, 2002, vol. II, p. 108.

(29) L'edizione delle Opere Postume uscì a Firenze con la falsa indicazione «Londra, 1804». Tra il 1805 e il novembre 1807 apparvero tredici volumi, che comprendevano le Satire, le Commedie, le traduzioni dal greco e dal latino, l'Abele, l'Alceste seconda e le Rime, nonché la Vita scritta da esso, consegnata ai due ultimi tomi e opportunamente emendata dalle critiche antifrancesi. Cfr. ivi, p. 109; Lettres inédites, cit., t. II, p. 195.

(30) Ivi, t. III, p. 56; p. 90. Cfr. ivi, t. II, p. 190.

(31) Ivi, t. II, p. 187; p. 193. Cfr. ivi, t. III, p. 54.

(32) Cfr. Editori italiani dell'Ottocento. Repertorio, a cura di A. Gigli Marchetti, M. Infelise, L. Mascilli Migliorini, M. I. Palazzolo, G. Turi, 2 voll., Milano, F. Angeli, 2004, vol. I, pp. 26-27 e 704-705; vol., II, p. 837 e p. 1002.

(33) Cfr. L.-G. PÉLIssier, Lettres et écrits, cit., 
nota nei suoi Souvenirs che la Stolberg gli aveva scritto nel 1784, in occasione del suo soggiorno a Baden, per organizzare un incontro, ma che poi questo non ebbe luogo ${ }^{34}$. Madame d'Albany rivide Bonstetten solo nel 1807, ossia trentatré anni dopo ${ }^{35}$. E lecito pensare che conoscenti comuni, quali lo stesso economista ginevrino o la baronessa de Staël, avessero contribuito a riannodare i contatti. Comunque la Contessa d'Albany fu lieta di rincontrarlo dopo tanto tempo e scrisse a Sismondi che lo aveva trovato «très aimable et très disposé à prendre le tems comme il vient. Sa philosophie est gaie et le rend indulgent» ${ }^{36}$.

Lettera del 23 giugno 1807

Florence le 23 juin $1807^{37}$

Jugez Monsieur du plaisir que vous m'avez procuré en voulant bien m'envoyer vôtre ${ }^{38}$ ouvrage $^{39}$ que j'ai recu dans le moment que ${ }^{40}$ je revenais de $\mathrm{M}$ [onsieu]r Moliny ${ }^{41}$ pour le charger de me le faire avoir. Une de mes amies a $a^{42}$ Gènève m'en avoit ${ }^{43}$ parlé avec tant d'éloges que je desirais vivamente ${ }^{44}$ de le lire. J'auroi un grand plaisir de ${ }^{45}$ vous

pp. 8-16; Italiam! Italiam! Ein neuentdecketer Karl Viktor von Bonstetten, Bern, Berlin, Frankfurt/M., Paris, Wien, Peter Lang, 1995 (tr. It. K. V. voN BONSTETTEN, Lettere dall'Italia, Locarno, Armando Dadò Editore, 1996), pp. 195-197; in Souvenirs de Charles Victor de Bonstetten, in Bonstettiana, Historisch-Kritische Ausgabe der Briefkorrispondenzen Karl Viktor von Bonstetten und seines Kreises (1753-1832), Bern, Peter Lang, 1996-, vol. I/1., p. 93. Si veda anche P. A. BorghegGiani, Les années difficiles de Bonstetten, in Ordre et caprice, cit., pp. 11-21.

(34) Souvenirs, cit., p. 93; L.-G. PÈLISSIER, Lettres inédites de la comtesse d'Albany, in Studi letterari e linguistici dedicati a Pio Rajna, Firenze, Tipografia E. Ariani, 1911, pp. 853-871.

(35) Souvenirs, cit., p. 93; J.-C.-L. Sismondi a Louise Stolberg, 12 agosto 1808, in Lettres inédites de J.C.L. Sismondi, cit., p. 74.

(36) C. Pellegrini, La contessa d'Albany, cit., p. 153; p. 281. L'impressione che ebbe Bonstetten nel rivedere la contessa non fu del tutto lusinghiera: «Heureusement le jour baissait; c'était bien sa voix, c'était un peu son regard, tout le reste était une vieille femme, que j'accusais dans mon cœur d'enfermer par magie celle que j'avais vue à Rome» (Souvenirs, cit., p. 93).

(37) «Archivio di Stato di Pescia» (d'ora in avanti ASP), Archivio Sismondi, Carteggio, b. 25 , n. 3. Sul retro: A Monsieur / Monsieur Leon Simonde / Sismondi / A Pescia / Toscana. Timbro postale: Firenze. Si può notare che nella data l'indicazione della città non è mai seguita dalla virgola; la Contessa d'Albany si discosta così dall'uso comune di allora. Si rileva pure un utilizzo assai ridotto degli accapo. I discorsi si susseguono senza soluzione di continuità, quasi si trattasse di un colloquio vis-à-vis con il destinatario. In questo senso si può parlare di style parlé nelle lettere di questa nobildonna.

(38) Forma antica della seconda persona plurale dell'aggettivo possessivo, derivata da vostre. Nonostante l'Académie française avesse introdotto, sin dal 1742, la distinzione attuale tra «votre» (aggettivo possessivo) e «vôtre» (pronome possessivo), la Contessa alterna le due forme. Cfr. M. Grevisse, $L e$ Bon Usage, éd. refondue par A. Goosse, Bruxelles, De Boeck, 2007, p. 784.

(39) J.-C.-L. SIsmONDI, Histoire des Républiques Italiennes, cit.

(40) Leggi: «au moment où». Locuzione, forse, calcata sull italiano.

(41) Giuseppe Molini (1772-1856) fu un noto libraio, editore-stampatore e bibliotecario fiorentino. Seguì la tradizione paterna, dedicandosi al commercio librario anche tramite una fitta rete di corrispondenti suoi familiari a Parigi e a Londra (cfr. Guida agli Archivi delle personalità della cultura in Toscana tra' 800 e '900. L'area fiorentina, a cura di E. Capannelli e E. Insabato, Firenze, Olschki, 1996, pp. 355-357).

(42) Leggi: «à». Si tratta di una variante molto frequente nella lingua della Contessa d'Albany, che ci limitiamo a segnalare solo in questa sua prima occorrenza. Da notare anche la frequente mancanza dell'accento circonflesso su tutte le vocali. Comunque l'uso disinvolto degli accenti non riguarda solo Louise Stolberg, ma è ampiamente documentato nelle corrispondenze dell'epoca. Un esempio autorevole è costituito dalle lettere di Madame de Staël. Su questo aspetto, cfr. Correspondance générale de Madame de Staël, texte établi et présenté par B. W. Jasinski et O. d'Haussonville, Genève, Slatkine (Réimpression), 2008-2009, t. I, p. LXXVI.

(43) La Contessa d'Albany non sposa completamente la riforma detta "volterriana", che prevedeva, tra l'altro, l'introduzione delle grafie in - $a i$ (soprattutto all'imperfetto indicativo e al condizionale presente), ma, come si può vedere, continua a usare spesso la grafia antica in -oi.

(44) Prestito occasionale.

(45) Leggi: «à». 
en remercier a votre retour a Florence. Celui a qui vous auriez voulu le présenter en auroit èté ${ }^{46}$ digne. Sa grande ame connoissoit le prix de la liberté, et ses ouvrages qui ne sont que l'interprete de ses sentimens ${ }^{47}$ le prouvent. Je ne conçois pas en vèrité comment une ame aussi sublime étoit nè ${ }^{48}$ dans des tems ${ }^{49}$ aussi corrompus et aussi peu dignes de lui et de ses écrits. J'ai eu toute la peine du monde de ${ }^{50}$ parvenir a finir d'imprimer ses ouvrages, a force d'obstination ${ }^{51}$ je suis quasi arrivee ${ }^{52}$ a la fin, et il ne manque plus que sa vie qui se reduit a deux volumes ${ }^{53}$. Les Comedies sont achevées mais il faut sçavoir ${ }^{54}$ si on les permettra a M[ilan] et cette capitale donnera le ton au reste de LItalie ${ }^{55}$ qui en cela comme dans tout le reste est dependante.

J'ai sçu l'aventure de M[a]d[am]e de Stäel mais j'ignorois quelle eut acheté une terre avec permission. Je suis ettonneè $e^{57}$ quelle s'obstine a vouloir aller dans un paÿs ${ }^{58} \mathrm{ou}^{59}$ elle éprouve tant de désagremens, il seroit plus noble d'avoir l'air de l'oublier ${ }^{60}$, elle a tant d'esprit, tant de ressource, et de renommée quelle n'a pas besoin de la futilité de la vie de Paris pour être heureuse; mais si elle avoit cette force de caractère unis a ces autres qualitées elle seroit audessus ${ }^{61}$ des autres mortalles ${ }^{62}$. Tout est compensé dans ce monde! Je n'ai connu qu'une seule personne qui reunissoit au geni ${ }^{63}$ une ame tendre, et forte, et une grande fermeté de caractère, vous devinez bien de qui je veux parler, d'après cela vous jugez bien que je le pleure tous les jours, et que dans ces momens funestes ou tout ce qui arrive, et nous entoure deplait, il seroit un appui pour

(46) Variante dell'accentuazione in -é. Non è raro incontrare, negli autografi della Contessa d'Albany, casi in cui queste ultime non sono accentate o recano un accento grave.

(47) Pierre Restaut, noto grammatico francese del 1700, raccomandava il mantenimento del grafe$\mathrm{ma}$ «t» al plurale dei nomi in -ant $\mathrm{e}$-ent (ad esempio, enfants, parents, moments), a scapito delle forme più antiche -an e -en (ad esempio, enfans, parens, momens). Cfr. Id., Principes généraux et raisonnés de la grammaire françoise, Paris 1732, pp. 410-411. La Principessa Stolberg usa entrambe le forme, con una marcata preferenza, però per quella più antica nelle lettere giovanili.

(48) L'Académie aveva formulato, sin dall'inizio del XVIII secolo, la regola attuale riguardo all'accordo del participio passato, anche se, sia nello scritto che nel parlato, essa tardò a imporsi. La Contessa d'Albany tende a fare l'accordo del participio passato in maniera corretta. Gli errori, a dire il vero abbastanza numerosi, potrebbero essere dovuti alla fretta o alla disattenzione. Sull'accordo dei participi, cfr. Nouvelle histoire de la langue française, sous la direction de J. Chaurand, Paris, Éditions du Seuil, 1999, pp. 331-335.

(49) «Tems» è attestato, in alternativa a «temps» (P. RICHELET, Dictionnaire de la langue françoise, ancienne et moderne, Lyon, chez P. Bruyset-Ponthus, t. 3, p. 699). Il Dictionnaire del 1740 riporta, invece, solo la voce temps (Dictionnaire de l'Académie française, Paris, J. B. Coignard, 1740, t. I, p. IX).

(50) Uso antico, poi sostituito da «avoir peine à» (Le Robert, Dictionnaire bistorique de la langue française, sous la direction d'A. Rey, Paris, Le Robert, 1998, t. II, p. 634).

(51) Scritto sotto (d'ora in poi s.): «de».

(52) Leggi: «arrivée».

(53) Riferimento alla Vita scritta da esso di Vittorio Alfieri, terminata nel maggio 1803 .

(54) Nelle lettere della Contessa questa forma compare spesso, accanto a «je scais» e «je sais». Si ricorda che le lettres étymologiques, o quantomeno quelle sentite come tali, erano state eliminate sin dalla terza edizione del Dictionnaire de l'Académie (1740). Cfr. N. Catach, Histoire de l'orthographe française, Paris, H. Champion, 2001, p. 37.

(55) Louise Stolberg non sempre rispetta le regole sull'apostrofo. Da notare, inoltre, l'uso peculiare e ricorrente di due lettere maiuscole, l'una davanti all'altra.

(56) Leggi: «qu'elle». I casi di mots collés sono frequenti nelle lettere. Da segnalare anche «la» (= «'a»).

(57) Leggi: «étonné». L'Académie française, nelle edizioni del Dictionnaire del 1740 e del 1762 , aveva auspicato lo scioglimento delle consonanti doppie (ad esempio «jeter», al posto di «jetter»; «apercevoir», invece di «appercevoir»), ma tale questione rimase in discussione fino alla fine del Settecento. La Stolberg, come molti suoi contemporanei, non rispetta molto questa raccomandazione. Cfr. N. CATACH, Histoire de l'orthographe, cit., pp. 233-236.

(58) Grafia antica, estremamente rara al tempo della Contessa, del sostantivo «pays» (Dictionnaire bistorique, cit., vol. II, p. 1407). Le grafie più comuni all'epoca erano: «pais» o «païs» (cfr. N. CATACH, Histoire de l'orthographe, cit., p. 246).

(59) Leggi: «où». L'uso dell'accento grave per il pronome relativo «où» faticava a imporsi ancora a metà Settecento (cfr. P. Restaut, Principes généraux, cit., p. 421). 506.

(60) Cfr. Mémoires de Mme de Staël, cit., pp. 505

(61) I casi di agglutinazione sono piuttosto numerosi nella corrispondenza di Madame d'Albany (ad esempio, «parceque», «aujourdhui», «audelà», «apresent», «apeine»).

(62) Leggi: «mortels».

(63) Leggi: «génie». 
mon ame, et pour mon esprit. M[a]d[am]e Moranda a èté la premiere a me parler de votre ouvrage elle m'a mandè ${ }^{64}$ aussi ${ }^{65}$ que M[onsieu] r de Sabran ${ }^{66}$ devoit m'apporter ${ }^{67}$ $\underline{\text { la Corinne }}^{68}$ mais jusqu'a présent je ne l'ai pas vù. J'ai écrit a Paris a Turin pour l'avoir mais finalement je ne l'ai pas, d'après cela vous me feriez un grand plaisir de charger le Proccacio de Lucques qui passe par chez vous de me l'apporter, je vous en ${ }^{69}$ seroit $^{70}$ très obligeé ${ }^{71}$. J'ai reçu une lettre de Rome de M[onsieu]r de Bonstetten qui m'annonce son arrivéè, mais comme depuis quattre ans il m'a déja mandé plusieurs fois de passer pour ${ }^{72}$ Florence, et qu'il a toujours pris l'autre chemin, je le croirai quand je le verrai. $\mathrm{Il} \mathrm{a}^{73}$ conservè un esprit, et une ame très jeune, et tres gaie malgré tous les mal'heurs qu'il a eprouvé $e^{74}$. C'est une chose très enviable!

Recevez de nouveau Monsieur tous mes remercimens de votre aimable attention comptez sur ma reconnaissance du plaisir que vôtre ouvrage certainement me procurera. Souvenez vous je vous prie de la promesse que vous $<$ me $>$ faites de venir a Florence cet èté j'aurai une véritable satisfaction de vous renouveller les sentimens que vos belles qualiteès m'ont inspirès Monsieur votre trés humble et obeissante Servante Louise de Stolberg Comtesse d'Albany

La seconda lettera si compone di quattro facciate. Come nel precedente inedito, l'indirizzo compare all'esterno e reca il sigillo della Contessa d'Albany su ceralacca rossa. Il testo dell'autografo occupa tre facciate: la prima conta ventitré righe, la seconda ventuno e la terza ventotto ${ }^{75}$.

I motivi d'interesse di questa lettera sono principalmente due: la discussione circa i futuri assetti politici dopo l'abdicazione di Napoleone, avvenuta il 16 aprile 1814, e la questione dell'indipendenza italiana. Si tratta di due nodi fondamentali che interessano da vicino, seppure per motivi diversi, sia la Stolberg che lo storico svizzero. La divergenza d'opinioni, come accennato, portò a una rottura quasi completa dei contatti epistolari nel 1815. La corrispondenza riprese negli anni successivi con la presa di coscienza da parte di Sismondi dell'irriducibilità della posizione antinapoleonica e legittimista della Contessa d'Albany, a cui fece da contraltare l'accettazione formale da parte di quest'ultima delle idee liberali del ginevrino.

L'inedito qui presentato contribuisce a far luce su quell'inquieto periodo della

(64) Il verbo «mander», nell'accezione di «faire savoir par message ou par lettre», è proprio dello stile epistolare classico (Dictionnaire bistorique, cit., t. II, p. 2116).

(65) S.: «qu».

(66) Elzéar-Louis-Marie, comte de Sabran (17741846), fu autore di pièces teatrali, tra cui Le Grand Monde, e di alcune opere di critica letteraria, come le Notes critiques, remarques et réflexions sur le 'Génie du christianisme' (1803), oltre che di poemi in versi, quali Repentir (1817), Ditbyrambe sur la mort de M. le duc de Berry et sur les dangers de l'Europe (1820). Fu esiliato da Napoleone e soggiornò a lungo a Coppet, tanto da essere considerato uno dei membri del gruppo. Cfr. P. Larousse, Grand dictionnaire universel du XIX siècle, ad vocem 'Sabran', Paris, Larousse, 1866-1876, t. 14, p. 17; M. DE RouGEMONT, L'activité théatrale dans te Groupe de Coppet: la dramaturgie et le jeu, dans AA.vV., Le Groupe de Coppet, Actes et documents du deuxième colloque de Coppet, Genève-Paris, Slatkine-Champion, 1977, p. 272; S. BALAYÉ, Madame de Staël. Ecrire, lutter, vivre, Genève, Droz, 1994, pp. 373-374.
(67) S.: «envoyer».

(68) La sottolineatura è nell'originale.

(69) $\mathrm{S}$.: «me».

(70) S.: «feroit».

(71) S.: «grand plaisir».

(72) L'uso di «pour» dopo un verbo di movimento per indicare una destinazione è piuttosto diffuso nel Settecento (cfr. Dictionnaire bistorique, cit., t. II, p. 2885).

(73) S.: «est».

(74) Negli anni immediatamente precedenti a questa lettera, Bonstetten aveva avuto problemi di salute ed era stato colpito da una serie di lutti, tra cui la morte della moglie, la perdita di un caro amico e la morte del secondo figlio (A. STEINLEN, Charles Victor de Bonstetten. Etude biographique et littéraire, Lausanne, G. Bridel Éditeur, 1860, pp. 254-258).

(75) Si precisa che, per mancanza di spazio nella terza facciata, la Contessa ha capovolto il foglio e ha aggiunto tre righe alle venti presenti nella prima facciata. 
loro amicizia. Madame d'Albany rimprovera a Sismondi l'opposizione troppo debole al regime napoleonico e continua a parlare di odio verso Bonaparte, anche dopo l'esilio all'Elba, auspicando il ritorno all'Ancien Régime ${ }^{76}$. La posizione dell'autore del Tableau de l'agriculture toscane nei confronti di Napoleone, invece, è più complessa. Pur non rinunciando al punto di vista mantenuto per tanti anni, lo storico cerca d'innalzarsi a una visione più alta, che trascenda il piano dell'astio e dell'accanimento. Egli inoltre prende in considerazione gli avversari dell'Imperatore, evidenziando come anch'essi non meritino stima, perché non si sono fatti portavoce di proposte alternative:

Il y a un homme pour lequel j'ai une forte aversion, qui n'a point changé; mais il n'y a pas un de ses adversaires pour lequel j'aie de l'affection ou de l'estime; et vraiment, ni dans les revers, ni dans les succès, ils n'ont rien fait pour la mériter. Cet homme a professé des principes qui me font bouillir le sang lorsque je les rencontre dans quelques écrits; mais ce n'est nullement à ces principes qu'en veulent ses adversaires; au contraire, c'est peut-être par là même qu'il a le plus de rapport avec eux. Son arrogance m'a été insupportable pendant de longues années; mais l'arrogance de ceux qui ont été si humbles pendant ces mêmes années me révolte peut-être encore plus ${ }^{77}$.

Queste parole, scritte proprio mentre l'esercito della sesta coalizione marciava su Parigi, non vennero comprese dalla Contessa d'Albany, che scambiò un simile atteggiamento per indecisione.

Sismondi ha una visione più ampia e pensa non solo alle conseguenze della caduta di Napoleone, ma prova una sincera inquietudine anche per l'avvenire dell'umanità. Non a caso lo storico apostrofò la Contessa con questa frase: «Vous regardez toujours le passé, moi je regarde toujours l'avenir». Oltre a ciò, in una lettera del 1 maggio 1814, osserva: «l'émotion d'une si grande catastrophe me fait un peu oublier le passé: le présent, l'avenir, remplissent bien plus ma pensée; je crains pour les plus forts l'ivresse du pouvoir» ${ }^{78}$. Il Sismondi, memore degli eccessi a cui era giunta durante il suo primo soggiorno in Toscana la reazione contro i francesi, teme di veder rinascere atteggiamenti demagogici, persino da parte di coloro che avrebbero dovuto rappresentare l'elemento più responsabile della popolazione. Constata tutto ciò anche nella cittadina dell'ex-Granducato dove viveva e si dice impensierito per la mancanza di elementi che possano costituire una nuova classe dirigente al posto di quella che stava tramontando. Il pensiero preoccupato dell'economista svizzero va agli amici in Francia e alle loro difficoltà ${ }^{79}$. Ciò che cerca di far capire alla Contessa d'Albany è la sua opposizione nei confronti della «toute-puissance partout où elle se trouve» ${ }^{80}$. Il timore è il ritorno al passato prerivoluzionario, quel passato, viceversa, tanto caro all'bôtesse di Firenze.

Tale paura, ovviamente, non viene per nulla condivisa da quest'ultima, la quale, nell'inedito sotto trascritto, sostiene che sono pochi i popoli che non meritano la schiavitù e aggiunge, non senza una punta di cinismo, di avere acquisito negli anni un grande disprezzo per gli uomini e per i governi. Secondo la Contessa i francesi, in particolare, non sono degni di nessuna indulgenza dal momento che, come scrive nella lettera del 10 maggio e in quella qui presentata, non hanno avuto il coraggio rovesciare Napoleone da soli, ma hanno ricercato l'aiuto delle nazioni straniere ${ }^{81}$. A

(76) Cfr. C. Pellegrini, La contessa d'Albany, cit., p. 334.

(77) J.-C.-L. Sismondi, Epistolario, cit., vol. I, p. 457.

(78) Lettres inédites de J.C.L. Sismondi, cit., p. 246.

(79) Ivi, p. 251; C. Pellegrini, La contessa d'Albany, cit., p. 208

(80) Lettres inédites de J.C.L. Sismondi, cit., p. 246.

(81) Cfr. C. Pellegrini, La contessa d'Albany, cit., p. 334 . 
sostegno delle sue affermazioni, la nobildonna di origine prussiana si richiama alla pubblicistica antinapoleonica e cita il pamphlet De Buonaparte et des Bourbons di Chateaubriand ${ }^{82}$, oltre che, nella parte finale dell'inedito, il Portrait d'Attila di Madame de Staël ${ }^{83}$.

La Stolberg non capisce il cambiamento d'idee che sta agitando Sismondi. Egli fu avversario di Napoleone in nome della libertà, quello stesso ideale di libertà che tanto ammirava in Alfieri, ma, da storico, cercò di osservare la realtà con pacatezza. Per questo riconobbe e apprezzò quanto Bonaparte intendeva realizzare nel campo del diritto o dell'educazione, diffondendo i risultati più fecondi della Rivoluzione francese $^{84}$. Alcuni fatti fecero sperare al letterato ginevrino che si potesse evitare un ritorno al passato. In particolare le sue illusioni furono riaccese dall'infelice esperienza del governo monarchico durante l'esilio all'isola d'Elba, dal coinvolgimento dell'amico Constant nella redazione della Costituzione liberale voluta da Napoleone durante i Cent-Jours, oltre che dal colloquio avuto dallo stesso Sismondi con quest'ultimo ${ }^{85}$. Tale cambiamento di Sismondi non fu perdonato dalla Contessa. Legata ancora al cosmopolitismo settecentesco, l'Albany non comprese il nuovo concetto di nazionalità che andava delineandosi nel gruppo di Coppet ${ }^{86}$.

Nella risposta alla lettera qui presentata, l'economista svizzero aggiunge alcune notizie proprio sull'animatrice del gruppo, vale a dire Madame de Staël. Questa si trovava ancora nella capitale inglese, ma si preparava a tornare in Francia, preceduta dall'edizione londinese di De l'Allemagne $e^{87}$. Sismondi si sentiva confortato dal fatto che anche l'amica scrittrice si dicesse estremamente rattristata dal vedere le truppe straniere in terra di Francia:

Dès que les Allemands ont passé le Rhin, elle n'a plus désiré que la paix avec Bonaparte, et elle a senti avec une profonde douleur l'humiliation de la France et de sa dépendance. Tout ressentiment personnel, toute haine, quelque motivée qu'elle fût, a cédé au sentiment fondamental d'une Française; elle n'a plus considéré que l'indépendance et l'honneur de la France. C'est ce sentiment national, Madame, que vous ne pouvez point partager et qui vous fait considérer d'un œil si différent des événements sur lesquels nous paraissions d'accord autrefois ${ }^{88}$.

Lo stesso autore dell'Histoire des Français si sentiva legato a quel paese da vincoli profondi. Tutte queste preoccupazioni, unite alla stanchezza di assistere ai grandi

(82) F.-R. DE ChateaubRiand, De Buonaparte et des Bourbons et de la nécessité de se rallier à nos princes légitimes, pour le bonheur de la France et celui de l'Europe, Paris, Arléa, 2004 (I ed. Paris, Mame Frères, 1814).

(83) G. De Stael-Holstein, Portrait d'Attila suivi d'une épitre à M. de Saint-Victor sur les sujets que le règne de Bonaparte offre à la poésie; par Louis-Aimé Martin, Paris, Librairie stéréotype, 1814. Cfr. I. A. Henning, L'Allemagne de Madame de Staël et la polémique romantique, Genève, Slatkine Reprints, 1975 (I ed. Paris, H. Champion, 1929), p. 6; Dictionnaire Napoléon, sous la direction de J. Tulard, Paris, Fayard, 1989, pp. 1592-1593; J. Tulard, Les vingt jours. Louis XVIII ou Napoléon?, Paris, $\mathrm{Fa}$ yard, 2001, p. 54.

(84) Cfr. C. Pellegrini, Il Sismondi e Napoleone, cit., p. 46.

(85) Sulla Costituzione elaborata da Constant, cfr. J. Tulard, Napoléon. Les grands moments d'un destin, Paris, Fayard, 2006, pp. 365-367.
(86) Cfr. C. Pellegrini, La contessa d'Albany, cit., p. 207; U. MarceluI, Il Gruppo de Coppet e il concetto di nazionalità, in AA.VV., Le Groupe de Coppet. Actes et documents du deuxième Colloque de Coppet, 10-13 juillet 1974, éd. par Simone Balaye [et al.], Genève-Paris, Slatkine-Champion, 1977, pp. 401-415; É. Hofmann, F. Rosset, Le groupe de Coppet: une constellation d'intellectuels européens, Lausanne, Presses Polytechniques et Universitaires Romandes, 2005; A. LAURENT, Le groupe de Coppet. Mythe et réalité. Staël, Constant, Sismondi, in AA.vv., Histoire du libéralisme en Europe, sous la direction de P. Nemo et J. Petitot, Paris, PUF, 2006, pp. 405-417; P. GARONNA, L'Europa di Coppet 17801820. Una lezione dalla storia per il futuro, Milano, F. Angeli, 2008.

(87) De l'Allemagne era già uscita nel 1810, ma era stata subito interdetta per ordine di Napoleone.

(88) Lettres inédites de J.-C.-L. Sismondi, cit., p. 255. 
cambiamenti della politica europea da quel piccolo angolo di Toscana, contribuirono a far maturare in lui la decisione di partire per Ginevra ${ }^{89}$.

L'altra questione affrontata nell'inedito della Contessa d'Albany è quella, già menzionata, dell'indipendenza italiana. Il suo giudizio è alquanto severo: essa sta più a cuore all'amico Sismondi che non agli italiani, tacciati, a seconda dei casi, di egoismo o di apatia. L'onore nazionale, scrive la Stolberg, passa in secondo piano rispetto al particolarismo territoriale.

Quest'ultima li accusa di mancanza di sentimento civile, quella stessa accusa che l'economista ginevrino, nel passo sopra riportato, rivolge alla hôtesse di Palazzo Gianfigliazzi. Sismondi concorda sul fatto che le possibilità d'indipendenza per l'Italia siano molto scarse e che sia quasi certo il ritorno dell'assolutismo monarchico, tant'è che osserva: «les espérances d'indépendance nationale s'évanouissent pour l'Italie, celles des limites à l'autorité absolue s'oublient» ${ }^{90}$.

$\mathrm{Ma}$ al centro della discussione continua a restare, per la Contessa, come risulta dalle lettere successive a quella qui esaminata, l'astio per Napoleone. Tanto più che in ciò trovava una fida alleata in un'altra amica comune a lei e alla Baronessa de Staël, vale a dire Madame Récamier ${ }^{91}$. Quest'ultima era stata ospite del salotto fiorentino e, nel momento in cui scrive la Stolberg, sta tornando a Parigi, dopo l'esilio comminatole da Bonaparte, a causa del suo salon parigino.

In sostanza, la lettera inedita della 'vedova' di Alfieri è in linea con le idee espresse in altre coeve indirizzate a Sismondi. La Contessa pensa che l'Austria non sia peggiore di altri occupanti e, sicuramente, mai peggiore del regime napoleonico. La sua visione politica è estremamente pessimistica: non è possibile conservare l'onestà quando si è a capo di una monarchia o si è tra i governanti di uno stato repubblicano. Il suo pragmatismo, l'attaccamento a certi privilegi Ancien Régime, unitamente al legame viscerale con il passato, in particolare al pensiero alfieriano di cui si proclamò sempre custode, le impedirono di modificare la sua posizione, tanto da farle dichiarare: «Il est inutile que nous discutions (...), car nous marchons par un chemin opposé, nous ne nous rencontrerons jamais» ${ }^{92}$.

\section{Lettera del 21 maggio 1814}

\section{Le 21 mai $[1814]^{93}$}

Je ne concois pas Mon cher Monsieur comment ${ }^{94}$ vous n'avez pas recu ma lettre du 10 partie le meme jour. Il y a un grand derangement dans les postes, on ne peut pas encore avoir des lettres de Paris. M[onsieu]r de Rohan Chabot ${ }^{95}$ est a Rome attendant

(89) Sismondi partì il 28 maggio 1814 (ivi, p. 251).

(90) J.-C.-L. Sismondi, Epistolario, cit., vol. II, p. 17.

(91) La Contessa d'Albany scrive il 4 giugno 1814 a Sismondi: «L'amie de Madame de Staël a été étonnée de sa générosité de désirer qu'on eût fait la paix avec le Tyran pour l'honneur national: elle n'est pas si héroïque» (C. Pellegrini, La contessa d'Albany, cit., p. 335). Su Juliette Récamier, cfr. F. Wagener, Madame Récamier, Paris, J.-C. Lattès, 1990. Vedi infra nota 106.

(92) La Contessa d'Albany aggiunge, a conclusione della medesima lettera: «Je regrette de differer d'opinions avec vous [Sismondi] parce que je crois que le miennes sont bonnes» (C. Pellegrini, La contessa d'Albany, cit., p. 336).

(93) ASP, Archivio Sismondi, Carteggio, b. 25, n. 4. Sul retro: «Al Signor Simond Sismondi / Pescia / Val Chiusa. Timbro postale: Firenze».

(94) L'uso di «comment» dopo il verbo «concevoir» è attestato nel Settecento, ma raro (F. BRUNOT, C. Bruneau, Précis de grammaire bistorique de la langue française, Paris, Masson, 1969, p. 490).

(95) Louis François Auguste de Rohan-Chabot (1788-1833), membro del potente casato di Rohan, fu ciambellano prima di Paolina Bonaparte, poi di Gioacchino Murat e, infine, di Napoleone. Mantenne tale carica fino all'esilio di quest'ultimo, nel 1814. Nel 1822 fu ordinato sacerdote e, nel 1830, 
le Pape ${ }^{96}$ il a passé l'hiver a Naples dans la societé de la Reine ${ }^{97}$ toujours en uniforme de chambellan de l'ex Empereur. Apeine les Bourbons ont ils étoient ${ }^{98}$ retablis qu'il a eté le premier a mettre la Coccarde $e^{99}$ blanche. Voilà les Français, et vous voulez qu'on les estime? et qu'on s'occupe de leur honneur nationale, cet honneur est cruellement lèse ${ }^{100}$ quand ils n'ont pas pù chasser leur Tyran, et seulement massacrer un Roi hônete, et qui n'avoit d'autre défaut que d'etre foible, et qu'il leur a fallu les armes des puissances coalisès pour les ${ }^{101}<$ des $>$ chainer. Lisez l'ouvrage de M[onsieu]r de Chateaubriant ${ }^{102}$ et la recapitulation qu'il fait de toutes les horreurs de ce monstre ${ }^{103}$, notre voisin, qui en dix ans a sacrifiés 5 millions de français, et 15 milliards de francs et plusque louis quatorze dans 77 ans de regne ${ }^{104}$. Quant a l'independance de LItalie vous etes bien bon de vous en occuper, quand ses habitans ne donnent pas un écus pour l'avoir. Ils sont trop egoiste, trop apathique pour faire le moindre sacrifice. Chaque petit paÿs veut avoir sa Capitale, et les habitans s'occupent peu de l'honneur nationale. Si le gouvernement Tyranique avoit encore duré dix ans peut etre qu'alors le malheur <les $>$ auroit reunis, encore j'en doute ? Il faut avouer aussi qu'il vaut encore mieux etre Toscan que de devenir province Napolitaine comme c'étoit la premiere ideé, car on voulait partager LItalie en deux portions. Peu de peuples Monsieur ne meritent pas d'etre esclaves vous etes bien bon de vous occuper d'eux. Pour moi l'experience m'a donnè le plus grand mepris pour les hommes, et les gouvernemens, et qui pis est on ne peut pas conserver l'honeteté en se melant des affaires d'ètat soit en commandant dans une republique ou dans une Monarchie. Il faut necessairement tromper $<$ les hommes et user de> moyens indignes d'un honete homme.

Il n'y a pas de doute l'autriche redevient puissante mais qu'importe ! Son gouvernement n'est pas pis que celui des autres, et le sera infiniment moins que celui des autres, et le sera infiniment moins que celui que nous venons d'avoir. Des le moment que LItalie ne peut, et ne veut pas devenir un Royame que ce soit l'autriche ou un autre Prince [?] qui la gouverne c'est assez indifferent. Je m'imagine que vous avez encore present l'affaire de Gillaume $[\ldots]^{105}$, et c'est ce qui vous donné de l'aversion pour cette Puissance; mais d'autres tems d'autres mœurs.

$\mathrm{M}[\mathrm{a}] \mathrm{d}[\mathrm{am}] \mathrm{e}$ Ricamier ${ }^{106}$ est a Rome et va retourner a Paris. Je doute que vous trouviez ici un compagnon de voyage. Il y a a Lucques $\mathrm{M}[\mathrm{a}] \mathrm{d}[\mathrm{am}] \mathrm{e}$ de la Tours ${ }^{107}$ qui veut aller a Toulouse, peut etre pourriez vous arranger ensemble.

Vous serez plus au courant des nouvelles ${ }^{108}$ a Genéve qu'ici. Il n'y a plus grand chose a savoir. Les Coalisés ${ }^{109}$ se retirent, les Souverains ${ }^{110}$ sont partis le onze pour Londres ${ }^{111}$

ricevette la porpora cardinalizia da Papa Pio VIII (T. Choffat, J.-M. Thiebaud, J. Tissot, Les comtois de Napoléon, cent destins au service de l'Empire, Yens sur Morges, Éditions Cabédita, 2006, pp. 233-234).

(96) Pio VII.

(97) Carolina Bonaparte, moglie di Gioacchino

Murat, re di Napoli, e sorella di Napoleone.

(98) Leggi: «été».

(99) Forma, forse italianizzata, di «cocarde».

(100) Leggi: «a été lésé».

(101) S.: «leur»

(102) F.-R. DE Chateaubriand, De Buonaparte et des Bourbons, cit. Vedi supra nota 82.

(103) La sottolineatura è nell'originale.

(104) La Contessa trae queste cifre dal pamphlet di Chateaubriand, De Buonaparte et des Bourbons, cit., p. 27.

(105) Lacuna non integrabile.

(106) Jeanne Françoise Julie Adélaïde Bernard, nota come Juliette Récamier o Madame Récamier (1777-1849) fu l'animatrice di un noto salotto tra
Direttorio e Primo Impero. Fu amica di Madame de Staël, di Benjamin Constant e di Chateaubriand. Cfr. F. Wagener, Madame Récamier, cit., p. 21 e passim.

(107) Henriette Lucy Dillon (1770-1853), nobildonna di origine irlandese, nota come Marchesa de La Tour du Pin, fu autrice di mémoires sulla fine dell'Ancien Régime, sulla Rivoluzione e l'Impero. Fu corrispondente, tra gli altri, di Madame de Staël (cfr. Marquise de La Tour du Pin, Mémoires, Mercure de France, Paris, 1979).

(108) S.: «ga»...

(109) Neologismo. Il participio passato «coalisé» figura nel Dictionnaire de l'Académie française del 1835. Madame de Staël lo usa per la prima volta in una lettera del 16 novembre 1790 (cfr. Correspondance générale, cit., t. I, p. 395).

(110) S.: $« R » .$.

(111) Prima dell'inizio del Congresso di Vienna, i sovrani vincitori e i loro ministri plenipotenziari si incontrarono a Londra. 
et voilà Louis dex huit qui va commencer sa tache difficille, il a trouvé une couronne d'epines et non pas une couronne de Roses. Probablement M[a]d[am]e de Stael sera retournée a Paris, elle a fait un paralelle d'Attilla et Napoleon ${ }^{112}$. Je trouve quelle lui fait bien de l'honneur. Portez vous bien et comptez a jamais sur mon tendre interet. Mille complimens a $\mathrm{M}[\mathrm{a}] \mathrm{d}[\mathrm{am}] \mathrm{e}$ votre mere $^{113}$. Je desirerais bien de vous revoir si vous faisiez votre voyage par Livo[ourn]e. Adieu. Donnez moi de vos nouvelles.

$<$ J'ignore la demeure de M[onsieu]r Foscolo à l'hotel Royale tout le monde le connait ${ }^{114}$. Il a eté a Boulogne et on ne lui a pas permis de venir ici. on ne sait pourquoi.>

FRANCESCA PISELLI

(112) G. De Stä̈l-Holstein, Portrait d'Attila, cit. Vedi supra nota 83.

(113) Henriette Esther Gabrielle Girodz (17481821). Notizie su di lei in F. Dal Degan, Sismondi nei diari della madre, 1792-1794 e 1809, Bologna, Patron, 2006, pp. 11-31.

(114) La Contessa d'Albany risponde a una precisa richiesta di Sismondi, formulata nella lettera del 15 maggio 1814. Il ginevrino, intenzionato a partire alla volta di Ginevra, spera di poter incontrare a Milano Ugo Foscolo, caro amico della Stolberg (cfr. Lettres inédites de J.-C.-L. Sismondi, cit., p. 253). Sui rapporti tra la Stolberg, Foscolo e Sismondi, cfr. C. Pellegrini, Ugo Foscolo, la contes- sa d'Albany e il Sismondi, in «Svizzera italiana», 61 (1947), pp. 33-38. Per quanto riguarda il carteggio tra la l'Albany e il poeta italiano, si rimanda a C. Antona-Traversi, D. Bianchini, Lettere inedite di Luigia Stolberg Contessa d'Albany a Ugo Foscolo e dell'abate Luigi di Breme alla Contessa d'Albany, Roma, Euseo Molino Editore, 1887; E. BertanA, La Contessa d'Albany e Ugo Foscolo, in «Giornale storico», XXXVIII, 1901, pp. 244-247; C. CoRDIÉ, Antonio Cicciaporci tra Guido Cavalcanti e Giulia Rinieri de' Rocchi. Con testimonianze dal carteggio della contessa d'Albany con gli amici senesi e con Ugo Foscolo, in «La Rassegna della Letteratura italiana», a. 94, n. 3, 1990, pp. 103-125. 